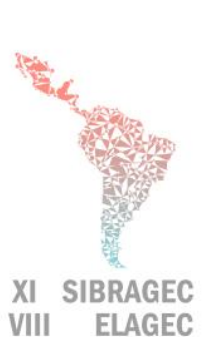

VIII ELAGEC

\section{SIMPÓSIO BRASILEIRO DE GESTÃO E ECONOMIA DA CONSTRUÇÃO \\ VIII ENCUENTRO LATINOAMERICANO DE GESTIÓN \\ Y ECONOMÍA DE LA CONSTRUCCIÓN}

Do conhecimento à ação: práticas avançadas de gestão da produção

Londrina, Paraná, Brasil. 23 a 25 de Outubro de 2019

\title{
ANÁLISE DO ÍNDICE DE VELOCIDADE DE VENDAS DO MERCADO IMOBILIÁRIO DE FORTALEZA SOB A ÓTICA DA PREVISÃO DE DEMANDA
}

\author{
MOREIRA, Felipe Fernandes (1). CAMPOS, Vanessa Ribeiro (2). \\ (1) Universidade Federal do Ceará, (85) 989248620, e-mail: felipef@gmail.com (2) Universidade Federal \\ do Ceará, e-mail: vanessa.campos@ufc.br.
}

\begin{abstract}
Demand forecasting is a key aspect of medium to long term investments, since such commitments are strongly dependent on how the present conditions will behave in the future. The objective of this paper is to study the sales velocity rate of the city of Fortaleza, Ceará, from the perspective of demand forecasting, more precisely by investigating the relationship between variables related to real estate public financing as well as the gross aggregated value of the construction national industry. Such analysis aligns with quantitative methods that aims at causality relationships among factors for forecasting demand. After subsequent data handling, the analysis was conducted by fitting trend lines with linear regression models that reveal with statistical significance correlation within dependent and independent variables. In conclusion, the analysis revealed time lags between the sales velocity rate and relevant economic data of the national real estate market, fact that can be employed on the development of forecasting models.
\end{abstract}

Keywords: Demand forecasting, real estate market, supply chain.

\section{INTRODUÇÃ̃O}

Vários são os autores que enfatizam a importância da previsão, especificamente a previsão de demanda, não só no planejamento e controle da produção, como fundamental para tomadas de decisões nos horizontes de curto, médio e longo prazo (CORREAA; CORRÊA, 2007; JACOBS; CHASE, 2018; SLACK; BRANDON-JONES; JOHNSTON, 2016). Por conta da inércia inerente ao processo de tomada de decisões (CORRÊA; CORRÊA, 2007) algumas exigem a antecipação de situações futuras, sendo necessário o emprego de métodos quantitativos e qualitativos de forma que ações sejam tomadas pela organização para concretizar oportunidades e proteger-se contra ameaças futuras.

Empreendimentos imobiliários se encaixam na categoria de investimentos de médio e longo prazo, com anos entre a decisão de compra do terreno e a entrega do produto (BALARINE, 2004). Além disso, são projetos que envolvem altos volumes financeiros e elevado risco (BALARINE, 2004; MAO; WU, 2011), frequentemente sujeitos a mudanças (OLSSON; SØRENSEN; LEIKVAM, 2015). Para Mao e Wu (2011) a escolha do investimento correto é mais importante que a gestão do empreendimento.

MOREIRA, F.F.; CAMPOS, V.R. Análise do índice de velocidade de vendas do mercado imobiliário de Fortaleza sob a ótica da previsão de demanda. In: SIMPÓSIO BRASILEIRO DE GESTÃO E 
Para investimentos de tal monta e de médio e longo prazo, métodos quantitativos que assumem a premissa de regularidade nas relações passadas para comportamentos futuros deixa de ser válida, sendo necessária a utilização de métodos qualitativos ou métodos quantitativos que buscam o relacionamento causal entre variáveis (CORREA; CORRÊA, 2007; SLACK; BRANDON-JONES; JOHNSTON, 2016). Previsões de longo prazo comumente estão relacionadas com decisões estratégicas que, apesar de arriscadas, demandam o conhecimento de variáveis a níveis agregados (CORRÊA; CORRÊA, 2007; JACOBS; CHASE, 2018).

O índice de velocidade de vendas (IVV) corresponde a uma medida do sucesso do empreendimento imobiliário (ILHA; HEINECK, 2000). No trabalho desenvolvido pelos autores, há uma análise do IVV a partir de características do empreendimento. Codes, Paixão e Campos (2017), também estudando dados do mercado de Fortaleza, Ceará, analisam a evolução de tal variável sob uma perspectiva geográfica.

Malizia (1991) desenvolveu um modelo de previsão de demanda para o mercado de imóveis comerciais do mercado metropolitano americano baseado em fundamentos econômicos, em particular dados do mercado de trabalho, atividade industrial, educação e mercado local. Zhang e Gao (2013) desenvolveram modelo para previsão de demanda do mercado imobiliário a partir de variáveis como PIB, taxa de juros do sistema de financiamento da habitação, volume de poupança e razão entre renda per capita e preço médio dos imóveis. Ambos recorreram à regressão linear como técnica para construção de modelos de previsão.

Os trabalhos citados abordam a previsão de demanda sob a ótica da estimativa do quão favorável o cenário econômico estará para certos investimentos. Este trabalho tem como objetivo de, através de modelos de regressão, explorar relacionamentos entre variáveis econômicas do mercado imobiliário e o índice de velocidade de vendas, parâmetro efetivamente utilizados em estudos de viabilidade de empreendimentos imobiliários. A partir daí, pretende-se desenvolver base para modelos de previsão de demanda aplicados ao mercado imobiliário.

\section{METODOLOGIA}

Primeiramente, foram coletados e compilados em planilha os dados do índice de velocidade de vendas (IVV) gerais para a cidade de Fortaleza calculado pelo SindusconCE e IEL/CE desde Janeiro de 2001 até Setembro de 2014 e complementados com dados calculados pelo Sinduscon-CE a partir de então até o fim do ano de 2016. O índice é calculado a partir da razão entre as vendas mensais sobre o total de unidades ofertadas - cálculo ilustrado na Equação 1.

$$
\operatorname{IVV}(\%)=\frac{\text { Vendas realizadas }}{\text { Oferta total de unidades }} \times 100
$$

Após a compilação, realizou-se uma análise exploratória dos dados e uma posterior análise de correlação com indicadores econômicos do mercado imobiliário nacional: volume de recursos do FGTS (Fundo de Garantia do Tempo de Serviço) a nível nacional aplicados no mercado imobiliário, recursos do SBPE (Sistema Brasileiro de Poupança e Empréstimo) concedidos para aquisição de imóveis no Ceará e o Valor Adicionado Bruto da Construção Civil nacional. 
SIBRAGEC - ELAGEC 2019 - de 23 a 25 de Outubro - LONDRINA - PR

\section{RESULTADOS}

A amostra de IVV's é composta por uma série com 165 observações, resultando numa média de $6,72 \%$ e desvio padrão de $3,40 \%$, o que corresponde a um coeficiente de variação de $50,3 \%$. Os dados apresentam obliquidade positiva $(1,640)$, mediana de $5,68 \%$ e a partir do histograma percebe-se moda da ordem de $5 \%$ ao mês. O máximo e mínimo da amostra são 20,65\% e 2,28\% respectivamente. Uma análise da evolução temporal da variáveis revela o impacto da crise econômica, apontado por Codes, Paixão e Campos (2017) em suas análises. A Figura 1 apresenta um histograma agrupando as medições mensais de IVVs da amostra, onde é possível observar forte assimetria para a direita.

Figura 1 - Histograma e curva cumulativa com os dados de IVV.

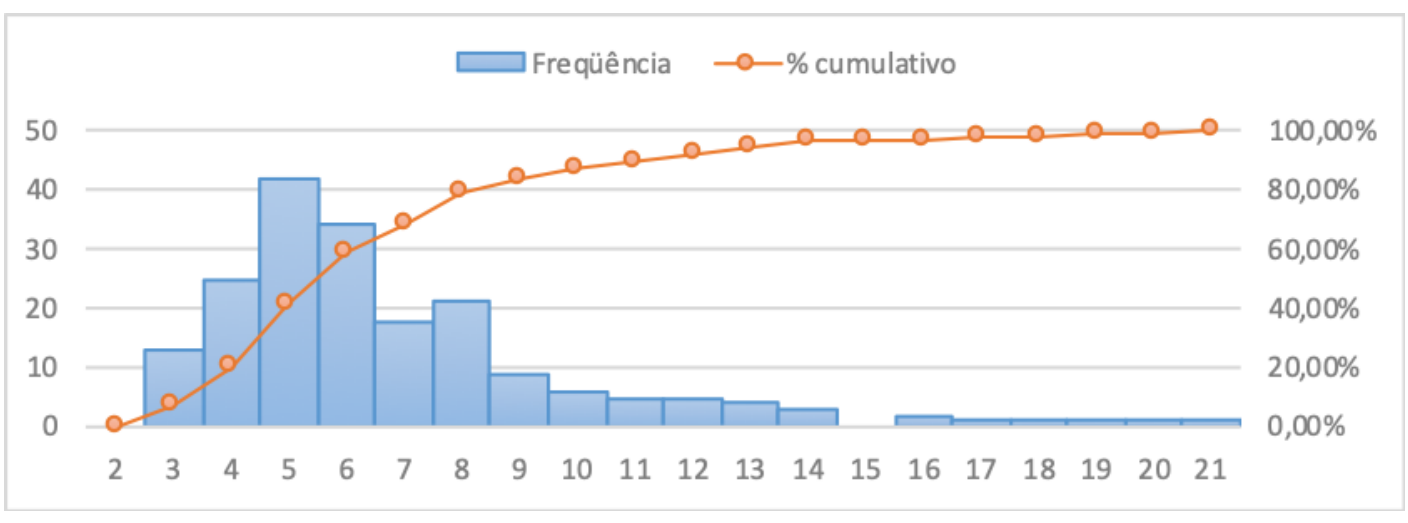

Fonte: Dados do Sinduscon-CE e IEL/CE, gráfico de elaboração própria.

Na Figura 2, em que a evolução temporal do índice é apresentada, é possível perceber quedas do IVV nos anos de 2014, 2015 e 2016 para patamares inferiores à média da amostra.

Figura 2 - Gráfico com a evolução temporal do IVV.

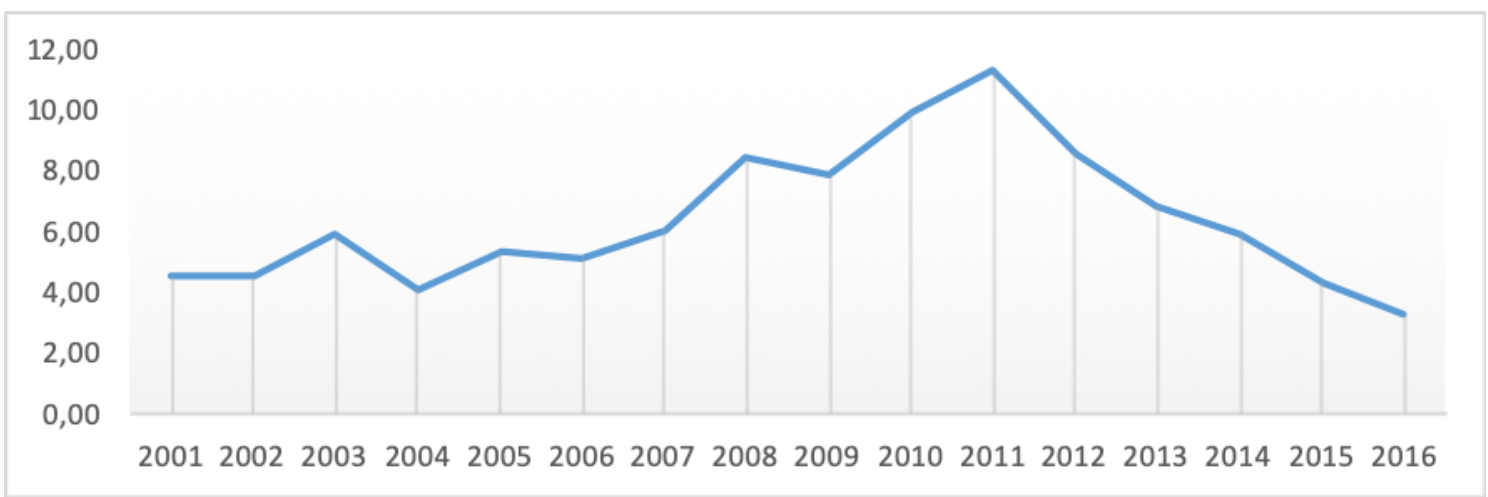

Fonte: Dados do Sinduscon-CE e IEL/CE, gráfico de elaboração própria.

Os dados referentes ao volume de financiamentos foram obtidos do banco de dados da Câmara Brasileira da Indústria da Construção (CBIC). Nesse banco, dados referentes ao FGTS e Valor Adicionado Bruto (VAB) são registrados em totais anuais, enquanto dados referentes ao SBPE são registrados mensalmente, sendo, portanto, necessário 
SIBRAGEC - ELAGEC 2019 - de 23 a 25 de Outubro - LONDRINA - PR

trabalhar a análise em bases anuais. As seguintes transformações foram aplicadas aos dados:

- IVV: Média mensal anual;

- FGTS: Volume anual em bilhões de reais;

- SBPE: Média mensal anual em milhões de reais;

- VAB: Volume anual em milhões de reais.

Inicialmente, correlações entre as variáveis foram avaliadas, não sendo possível detectar relacionamentos fortes - a correlação entre o IVV e FGTS, SBPE e VAB foram 0,041, 0,136 e 0,211 , respectivamente, correlações positivas ainda que fracas. Porém, a partir de uma visualização gráfica do comportamento das variáveis com o tempo, foi possível visualizar tendências (Figura 3, Figura 4 e Figura 5).

\section{Figura 3 - Gráfico com evolução do FGTS e IVV.}

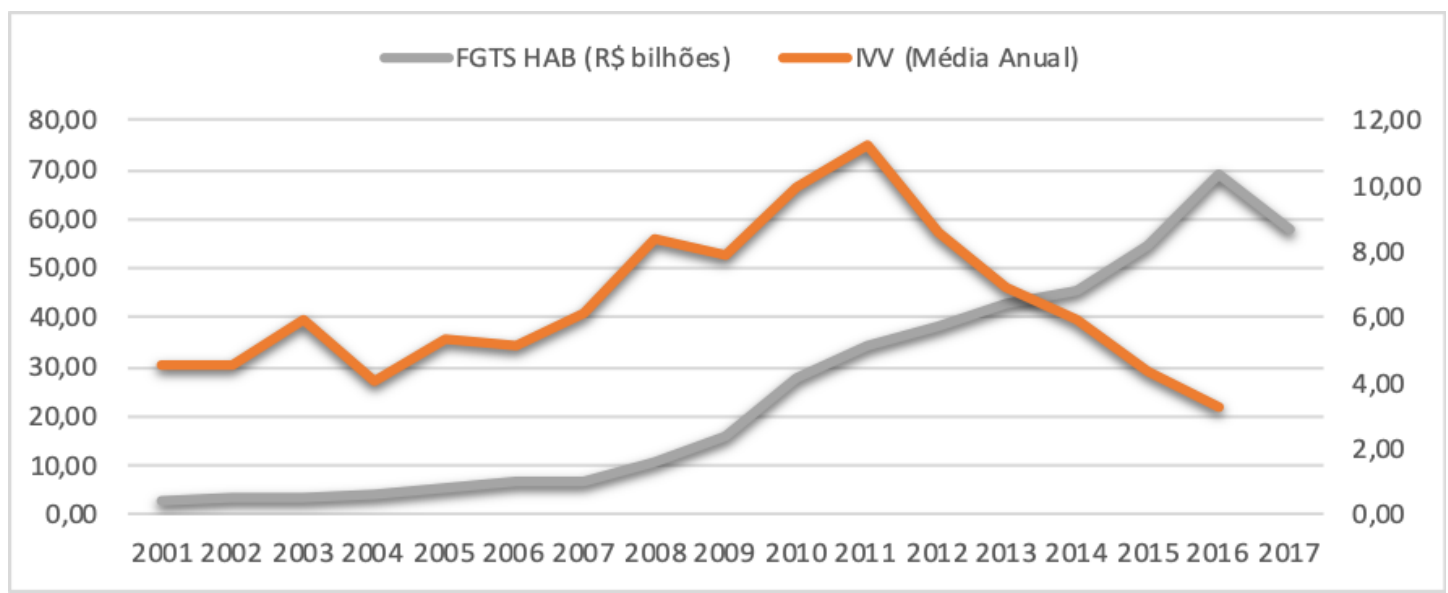

Fonte: Dados da CBIC, Sinduscon-CE e IEL/CE, gráfico de elaboração própria.

Figura 4 - Gráfico com evolução do SBPE e IVV.

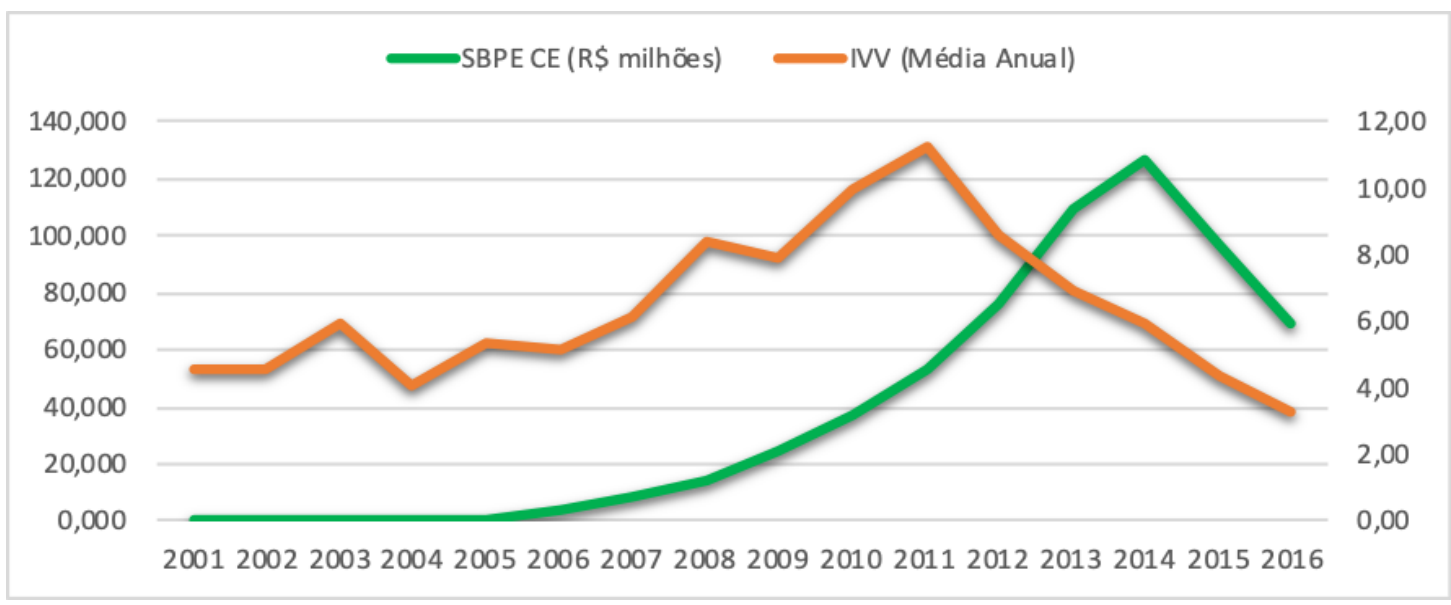

Fonte: Dados da CBIC, Sinduscon-CE e IEL/CE, gráfico de elaboração própria. 
SIBRAGEC - ELAGEC 2019 - de 23 a 25 de Outubro - LONDRINA - PR

Figura 5 - Gráfico com evolução do VAB e IVV.

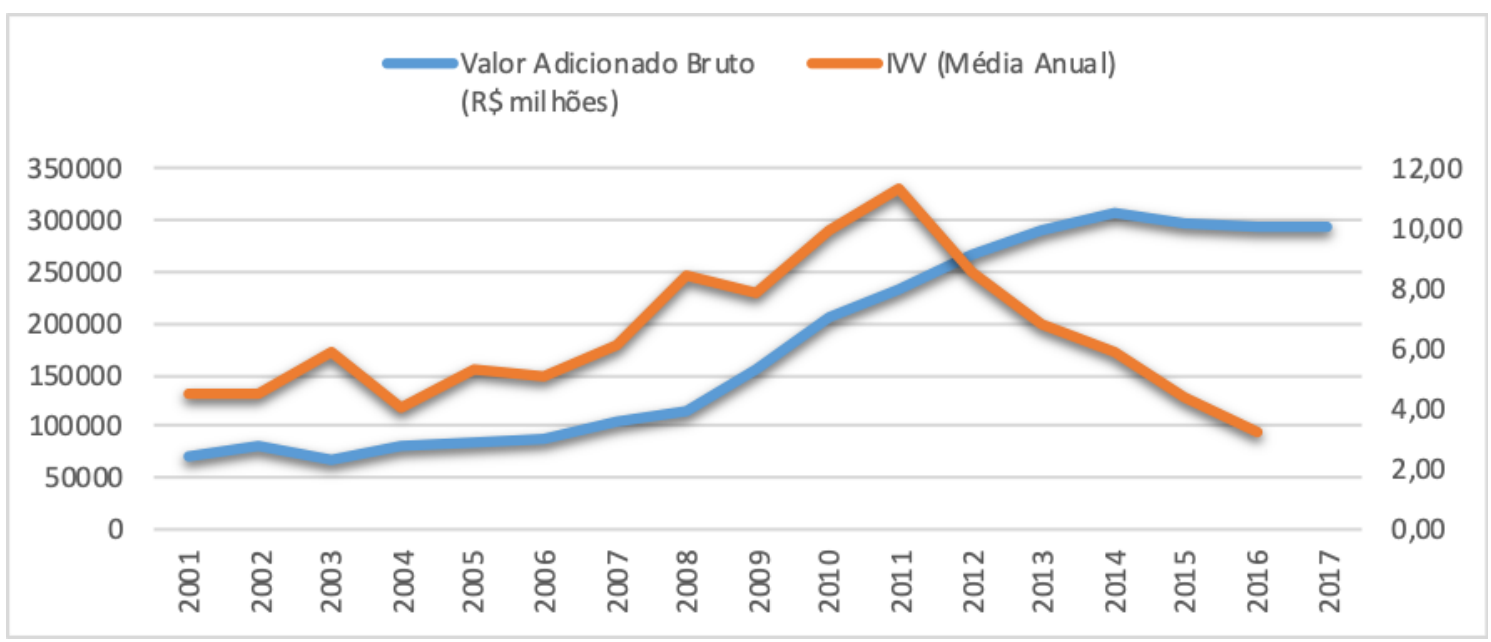

Fonte: Dados da CBIC, Sinduscon-CE e IEL/CE, gráfico de elaboração própria.

A partir da Figura 3 e Figura 4, é possível perceber que quedas no IVV não acarretam quedas em FGTS e SBPE, o que pode ser explicado pelo caráter de longo prazo apontado por Balarine (2004). De fato, há uma um período de tempo entre o início da comercialização das unidades e sua entrega de fato, quando são contratados financiamentos. Observa-se que para o FGTS, a defasagem é da ordem de 5 anos, enquanto que para o SBPE a defasagem é de 3 anos. Tal disparidade pode ser explicada pelo fato de que FGTS agrupa dados do mercado nacional, enquanto o SBPE reúne dados referentes ao mercado cearense, mais semelhante ao âmbito do IVV, que é medido para Fortaleza.

Porém, afora o período de crise, há uma correlação importante entre tais variáveis. Considerando os anos de 2013 a 2016 como anos de crise (já que são os anos em que IVV caminha para patamares abaixo da média da série), ou seja, utilizando apenas dados de 2001 até 2013, encontra-se correlações de 0,868 e 0,809 para IVV e FGTS e SBPE respectivamente. Ainda, ao incluir uma variável aleatória que indica a incidência de crise nos anos de 2013 a 2016, é possível realizar uma regressão linear com níveis de significância superiores a 95\%. O Tabela 1 apresenta tais resultados. É possível perceber que a regressão com a variável SBPE fornece menor erro padrão e maior coeficiente de determinação $\left(\mathrm{R}^{2}\right)$.

Tabela 1 - Resultados da Regressão Linear

\begin{tabular}{|c|c|c|c|c|c|c|}
\hline Variável & Interseção & Coeficiente & Crise & p-valor & $\begin{array}{c}\text { Erro } \\
\text { padrão }\end{array}$ & R2 \\
\hline $\begin{array}{c}\text { FGTS Imob. } \\
\text { (R\$ bilhões) }\end{array}$ & 5,41 & 0,10 & $-5,85$ & $\begin{array}{c}\text { Coef.: } 0,02 \\
\text { Crise: } 0,01\end{array}$ & 1,85 & 0,4257 \\
\hline $\begin{array}{c}\text { SBPE - Ceará } \\
\text { (R \$ milhões) }\end{array}$ & 5,49 & 0,07 & $-7,64$ & $\begin{array}{c}\text { Coef.: }<0,01 \\
\text { Crise: }<0,01\end{array}$ & 1,36 & 0,6922 \\
\hline
\end{tabular}

Fonte: Elaboração própria.

Já para o relacionamento do VAB com o IVV, uma considerável correlação positiva é obtida quando se analisa o IVV com a variação anual do VAB (correlação igual a 
0,464), sendo ela mais proeminente quando se trabalha com uma defasagem entre as variáveis, por exemplo, combinando a variação de 2001 do VAB com o IVV de 2002 e assim por diante (correlação igual a 0,818). A Figura 6 apresenta um diagrama de dispersão ilustrando a correlação positiva entre ambas variáveis.

\section{Figura 6 - Correlação positiva entre IVV (eixo vertical) e variação anual percentual do VAB (eixo horizontal).}

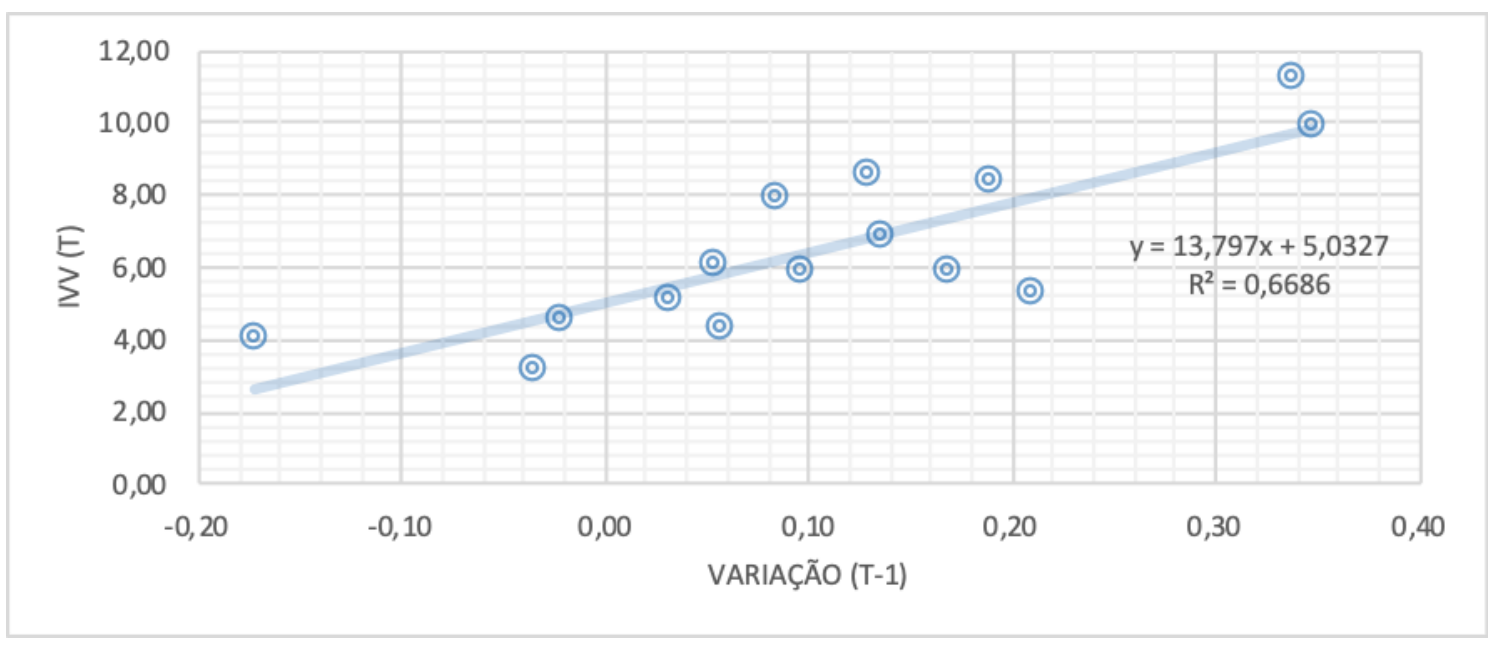

Fonte: Dados da CBIC, Sinduscon-CE e IEL/CE, gráfico de elaboração própria.

Os resultados da regressão linear entre as duas variáveis são apresentados no Tabela 2. Os resultados são comparáveis aos obtidos para a regressão entre SBPE e IVV no Tabela 1, sendo os anteriores ligeiramente mais precisos (menor erro padrão e maior coeficiente de determinação).

Tabela 2 - Resultados da Regressão Linear com VAB defasado em um ano.

\begin{tabular}{|c|c|c|c|c|c|}
\hline Variável & Interseção & Coeficiente & p-valor & Erro padrão & $\mathbf{R}^{2}$ \\
\hline $\begin{array}{c}\text { Variação (\%) do } \\
\text { VAB Defasado } \\
\text { (t-1) }\end{array}$ & 5,03 & 13,78 & $<0,01$ & 1,37 & 0,6686 \\
\hline
\end{tabular}

Fonte: Elaboração própria.

\section{CONCLUSÕES}

Os resultados da análise mostram que é possível inferir sobre o Índice de Velocidade de Vendas a partir das variáveis econômicas do mercado imobiliário nacional. Foi possível detectar que as variáveis relacionadas aos volumes de financiamento são impactadas por conta de situações adversas no mercado imobiliário com certa defasagem com significância estatística.

No caso, os volumes de financiamento do SBPE alocados para aquisição de imóveis para o Ceará seguiram a grande tendência de queda do IVV após 3 anos, um período compatível com a duração entre o lançamento de um empreendimento e sua entrega. Para a variável de volume financeiro do FGTS alocado no mercado imobiliário, a defasagem encontrada foi de 4 anos. 


\section{SIBRAGEC - ELAGEC 2019 - de 23 a 25 de Outubro - LONDRINA - PR}

Com os modelos de regressão foi possível isolar o forte impacto da crise no IVV e aferir a forte correlação entre as variáveis. Em cenários econômicos estáveis, ambas variáveis podem oferecer estimativas do IVV no ano corrente.

Com o modelo obtido pela regressão linear entre o IVV e a variação do Volume Agregado Bruto da construção civil, por conta da defasagem aplicada, pode-se obter a partir de dados de um certo ano previsões para o IVV no ano seguinte - ou seja com os dados de variação para o ano de 2018, poder-se-ia prever o valor do IVV de 2019, construindo um intervalo de confiança com os erros apresentados pelo modelo.

Os resultados são consistentes com o encontrado na bibliografia. As variáveis dependentes SBPE e FGTS estão relacionadas ao sistema de financiamento da habitação, tal qual as variáveis taxa de juros e poupança utilizadas por Zhang e Gao (2013). Da mesma forma, a variável VAB relaciona-se com a atividade industrial, tal como estudo por Malizia (1991).

Como continuidade ao estudo, propõe-se utilizar variáveis econômicas para a previsão do índice de velocidade de vendas futuros e comparação com dados reais observados, bem como a utilização de modelos de séries temporais.

\section{REFERÊNCIAS}

BALARINE, O. F. O. O uso da análise de investimentos em incorporações imobiliárias. Production, v. 14, n. 2, p. 47-57, 2004.

CODES, B. N.; PAIXÃO, E. A.; CAMPOS, V. R. Análise do índice de velocidade de vendas dos imóveis da cidade de Fortaleza. In: VII Congresso Brasileiro de Engenharia de Produção. Anais... 2017

CORRÊA, C. A.; CORRÊA, H. L. Administração da Produção e Operações: uma abordagem estratégica. 2. ed. [s.1.] Atlas, 2007.

ILHA, J. C. G.; HEINECK, L. F. Análise Da Oferta Imobiliária E Índices De Velocidade Vendas: Um estudo para o mercado de Florianópolis. In: Encontro Nacional de Tecnologia do Ambiente Construído - ENTAC. Anais... 2000

JACOBS, F. R.; CHASE, R. B. Operations and Supply Chain Management. 15. ed. New York: McGraw Hill, 2018.

MALIZIA, E. E. Forecasting Demand for Commercial Real Estate Based on the Economic Fundamentals of U.S. Metro Markets. Journal of Real Estate Research, v. 6, n. 3, p. 251-266, 1991.

MAO, Y.; WU, W. Fuzzy Real Option Evaluation of Real Estate Project Based on Risk

Analysis. Systems Engineering Procedia, v. 1, n. 1, p. 228-235, 2011.

OLSSON, N. O. E.; SØRENSEN, A.; LEIKVAM, G. On the Need for Iterative Real Estate

Project Models - Applying Agile Methods in Real Estate Developments. Procedia

Economics and Finance, v. 21, n. 1, p. 524-531, 2015.

SLACK, N.; BRANDON-JONES, A.; JOHNSTON, R. Operations Management. 8. ed. [s.l.] Pearson Education, 2016.

ZHANG, B.; GAO, X. Demand Forecasting Analysis of Xi' an Real Estates. In: International Conference on Construction and Real Estate Management 2013, Karlsruhe, Germany. Anais... Karlsruhe, Germany: 2013. 